\title{
Beat Rate Variability in Murine Embryonic Stem Cell-Derived Cardiomyocytes: Effect of Antiarrhythmic Drugs
}

\author{
Julius Niehoffa,b Matthias Matzkies ${ }^{\mathrm{b}} \quad$ Filomain Nguemo $^{\mathrm{b}} \quad$ Jürgen Hescheler ${ }^{\mathrm{b}}$ \\ Michael Reppela,b \\ aDepartment of Cardiology, University of Lübeck, Lübeck, 'DInstitute of Neurophysiology, University of \\ Cologne, Cologne, Germany
}

\section{Key Words}

Beat Rate Variability • Antiarrhythmic Drugs • Microelectrode Array • Murine Embryonic Stem Cells $•$ Cardiomyocytes

\begin{abstract}
Background/Aims: Heart rate variability (HRV) refers to the fluctuation of the time interval between consecutive heartbeats in humans. It has recently been discovered that cardiomyocytes derived from human embryonic and induced pluripotent stem cells show beat rate variability (BRV) that is similar to the HRV in humans. In the present study, clinical aspects of HRV were transferred to an in vitro model. The aims of the study were to explore the BRV in murine embryonic stem cell (mESC)-derived cardiomyocytes and to demonstrate the influence of antiarrhythmic drugs on BRV as has been shown in clinical trials previously. Methods: The Microelectrode Array (MEA) technique was used to perform short-term recordings of extracellular field potentials (FPs) of spontaneously beating cardiomyocytes derived from mESCs (D3 cell line, aPig-44). Offline analysis was focused on time domain and nonlinear methods. Results: The Poincaré-Plot analysis of measurements without pharmacological intervention revealed that three different shapes of scatter plots occurred most frequently. Comparable shapes have been described in clinical studies before. The antiarrhythmic drugs Ivabradine, Verapamil and Sotalol augmented BRV, whereas Flecainide decreased BRV parameters at low concentrations (SDSD $79.0 \pm 8.7 \%$ of control at $10^{-9} \mathrm{M}, \mathrm{p}<0.05$ ) and increased variability measures at higher concentrations (SDNN $258.8 \pm 42.7 \%$ of control at $\left.10^{-5} \mathrm{M}, \mathrm{p}<0.05\right)$. Amiodarone and Metoprolol did not alter BRV significantly. Conclusions: Spontaneously beating cardiomyocytes derived from mESCs showed BRV that appears to be similar to the HRV known from humans. Antiarrhythmic drugs affected BRV parameters similar to clinical observations. Therefore, our study demonstrates that this in vitro model can contribute to a better understanding of electrophysiological properties of $\mathrm{mESC}$-derived cardiomyocytes and might serve as a valuable tool for drug safety screening.
\end{abstract}




\section{Cellular Physiology Cell Physiol Biochem 2016;38:646-658 \begin{tabular}{l|l|l|l} 
DOI: 10.1159/000438657 & (c) 2016 The Author(s). Published by S. Karger AG, Basel \\
www.karger.com/cpb
\end{tabular} \\ Niehoff et al.: Effect of Antiarrhythmic Drugs on Beat Rate Variability}

\section{Introduction}

Ever since the electrophysiological properties of spontaneous beating clusters of cardiomyocytes derived from embryonic stem cells (ESCs) were examined, various characteristics of the human heart have been demonstrated to be similar in this in vitro model. Not only basic electrophysiological properties such as stable spontaneous beating frequency, but also the response to cardioactive drugs was similar in electrocardiogram (ECG)-like recordings of extracellular field potentials (FPs) that have been the subject of in vitro studies [1-4]. In line with these approaches, it has recently been demonstrated that ESC-derived cardiomyocytes show beat rate variability (BRV) that is similar to heart rate variability (HRV) in humans [5, 6].

HRV describes the physiological fluctuations of the instantaneous heart rate and the physiological oscillation of the interval between consecutive heartbeats in humans [7]. It is mainly the expression of the sustained impact of the autonomic nervous system on the heart rate. Vagal and sympathetic signals continuously mediate the influence of endogenous and exogenous stimuli on HRV. Among others, respiration, blood pressure regulation and thermoregulation are capable of modifying the inter-beat interval (IBI) [8-10]. The HRV is also affected by certain cardiac and non-cardiac specific diseases. In clinical practice, the analysis of the HRV is applied to estimate the function of the autonomic nervous system and has become an important prognostic tool. It has been shown in clinical studies that depressed variability, for instance, can be a predictor of short- as well as long-term mortality after acute myocardial infarction [11, 12]. Furthermore, a study that focused on patients with chronic congestive heart failure could demonstrate that per 10 ms increase of SDNN the risk of mortality decreased by $20 \%$ [13]. Modulation of HRV, for example by pharmacological intervention, became the focus of clinical trials. Several studies investigated the effects of antiarrhythmic drugs on HRV in diseased as well as healthy individuals. Ivabradine, Verapamil and Sotalol, for instance, have been reported to increase HRV parameters in patient groups suffering from various cardiac diseases [14-16]. In contrast, Flecainide has been found to decrease HRV parameters in patients after acute myocardial infarction as well as in patients without structural heart disease $[17,18]$. Other antiarrhythmic drugs, such as Amiodarone, do not induce any essential changes in standard HRV parameters [19]. There are also drugs that affect time domain measures of HRV inconsistently. Depending on the patient group, $\beta$-blockers can increase a single HRV parameter, whereas other time domain indexes are not affected [20]. Detailed information about the effects of antiarrhythmic drugs on time domain parameters of HRV is summarized in Table 1.

In our study, we focused on the clinical aspects of HRV and transferred this knowledge to a specific in vitro model, using spontaneously contracting cardiomyocytes derived from murine embryonic stem cells (mESCs) in combination with Microelectrode Arrays (MEAs) and standard antiarrhythmic drugs. As a first approach, we found that mESCs-derived cardiomyocytes are eligible for this study, due to the high sensitivity to antiarrhythmic substances that has been reported previously by our working group [2]. The MEA technique allows to measure extracellular FPs with high temporal and spatial resolution and has not only contributed to the exploration of electrophysiological properties of contracting cardiomyocytes derived from ESCs in previous studies, but has also proved its potential to serve as an important tool for preclinical drug safety screening [2, 3, 5]. Nevertheless, whether the effect of drugs on HRV known from in vivo studies, is similar to the effect on BRV in vitro, is still unclear. The investigation of this question would contribute significantly to a better understanding of the value of this model for preclinical drug testing as well as the comparability of HRV and BRV. Primarily, we focused on time domain methods as standard and well-established analysis of HRV to describe the effects of pharmacological intervention on BRV. In addition, we used a nonlinear method (Poincaré-Plots) as supplemental tool for BRV analysis. 


\section{Cellular Physiology Cell Physiol Biochem 2016;38:646-658 \begin{tabular}{l|l|l|}
\hline DOI: 10.1159/000438657 & C 2016 The Author(s). Published by S. Karger AG, Basel \\
\hline
\end{tabular} \\ Niehoff et al.: Effect of Antiarrhythmic Drugs on Beat Rate Variability}

Table 1. Effect of antiarrhythmic drugs on time domain parameters of HRV in clinical studies. $\uparrow=$ increase; $\downarrow=$ decrease; $(\downarrow)=$ trend towards reduction, $\leftrightarrow=$ unchanged; SDNN= standard deviation of normal-tonormal intervals; RMSSD = square root of the mean squared differences of successive normal-to-normal intervals; NN50= number of interval differences of successive normal-to-normal intervals exceeding 50ms; pNN50 $=$ NN50 divided by the total number of normal-to-normal intervals

\begin{tabular}{clcccc}
\hline \multirow{2}{*}{ Drug } & \multicolumn{1}{c}{ Patient Group } & \multicolumn{3}{c}{ Observed Time Domain Parameters } \\
& \multicolumn{1}{c}{ SDNN } & RMSSD & NN50 & pNN50 \\
\hline Ivabradine & Nonischemic heart failure [15] & $\uparrow$ & $\uparrow$ & & $\uparrow$ \\
Verapamil & Acute myocardial infarction [14, 21] & $\uparrow$ & $\uparrow$ & & $\uparrow$ \\
Sotalol & Ventricular arrhythmia [16] & $\uparrow$ & $\uparrow$ & & $\uparrow$ \\
Amiodarone & Ventricular arrhythmia [19] & & & $\leftrightarrow$ & \\
& Congestive heart failure [22] & $\leftrightarrow$ & $(\downarrow)$ & & $\downarrow$ \\
& No structural heart disease [18] & $\downarrow$ & $\downarrow$ & & $\downarrow$ \\
Flecainide & Ventricular arrhythmia [19] & & & $\downarrow$ & \\
& Acute myocardial infarction [17] & & $\downarrow$ & $\downarrow$ & $\downarrow$ \\
& Decompensated heart failure [23] & $\uparrow$ & $\uparrow$ & & $\uparrow$ \\
$\beta$ & Acute myocardial infarction [20] & $\uparrow$ & $\leftrightarrow$ & & $\leftrightarrow$ \\
& Remote myocardial infarction [24] & $\leftrightarrow$ & $\uparrow$ & & $\uparrow$ \\
& Healthy persons [25] & & $\uparrow$ & & $\uparrow$ \\
\hline
\end{tabular}

In the present study, we found that BRV was present in murine ESC-derived cardiomyocytes. In addition, we demonstrated that the effects of standard antiarrhythmic drugs on BRV were similar to the effects on HRV in humans.

\section{Material and Methods}

\section{Cell Culture}

Murine ESCs of the line D3 [26, 27] ( $\alpha$ Pig-44) were co-cultured with a layer of fibroblast feeder cells in culture medium consisting of Iscove's Modified Dulbecco's Medium (IMDM) supplemented with fetal bovine serum (FBS, 15\%), non-essential amino acids (NEA, 1X), 2-Mercaptoethanol (50 $\mu \mathrm{M}$ ), leukemia inhibitory factor (LIF, $1 \mathrm{U} / \mu \mathrm{l}, \mathrm{ESGRO}^{\circledR}$ Merck Millipore) and Geniticin $(0.5 \mathrm{mg} / \mathrm{ml})$. The mESCs were passaged three times per week.

\section{Cell Differentiation}

In order to differentiate mESC into embroid bodies (EBs), $10^{6}$ enzymatically dissociated mESCs were cultured on a horizontal shaker for two days in differentiation medium consisting of IMDM supplemented with FBS (20\%), NEA (1X), 2-Mercaptoethanol $(100 \mu \mathrm{M})$ and Penicillin combined with Streptomycin (100 $\mathrm{U} / \mathrm{ml}$ ). On day $2,10^{3}$ EBs were transferred to separate bacterial dishes with fresh differentiation medium and placed on a horizontal shaker for further differentiation. The medium was exchanged on day 7 for the first time. The first beating EBs could usually be detected on day 8 of differentiation. If not stated otherwise, all media and reagents mentioned above were purchased from Gibco ${ }^{\circledR}$ (Invitrogen, Karlsruhe, Germany).

\section{Experimental Setup}

Extracellular FPs were recorded using MEAs in combination with the MEA1060-Inv-BC amplifier and data acquisition system from Multichannel Systems (MCS, Reutlingen, Germany) [28]. The standard MEA chambers consisted of an internal reference electrode and 60 titanium nitride-coated gold electrodes with a diameter of $30 \mu \mathrm{m}$ each. The electrodes were arranged in an 8x8 grid with an inter-electrode distance of 200 $\mu \mathrm{m}$. Measurements were performed with a sampling rate of $2 \mathrm{kHz}$. A perfusion system was established using a customized perfusor (B. Braun Melsungen AG, Germany) to ensure a continuous flow of $500 \mu \mathrm{l}$ per minute 


\section{Cellular Physiology Cell Physiol Biochem 2016;38:646-658 \begin{tabular}{l|l|l|l|l} 
DOI: 10.1159/000438657 2016 The Author(s). Published by S. Karger AG, Basel & $\begin{array}{l}\text { C) } \\
\text { www.karger.com/cpb }\end{array}$
\end{tabular} \\ Niehoff et al.: Effect of Antiarrhythmic Drugs on Beat Rate Variability}

and a constant concentration of the pharmacological compound within the MEA chamber. Temperature was kept constant at $37^{\circ} \mathrm{C}$ with a heating plate as well as a heating cannula (PH01, MCS). Both were controlled automatically by a temperature controller (TC 02, MCS).

\section{Experimental Procedure}

MEA chambers were prepared by coating the electrode area with $0.1 \%$ gelatine and fibronectine (both Sigma Aldrich, Germany). Spontaneously beating EBs were usually selected on day 10 of differentiation and placed manually onto the electrode area. The EBs were kept under incubator conditions for 48 hours in order to ensure sufficient tissue attachment onto the electrodes. Consequently, all measurements were performed during the early or intermediate stage of development [29]. Prior to the experiment, the differentiation medium was replaced by IMDM. Additionally, the amount of medium was raised to $500 \mu \mathrm{l}$. Experiments always started with the recording of a control measurement, followed by measurements with one of the pharmacological substances. The concentration of the drug was increased after each recording. The standard recording time period for each concentration was eight minutes. As final step, a washout was performed.

\section{Antiarrhythmic Substances}

The following antiarrhythmic substances were tested (all from Sigma Aldrich, Germany): Ivabradine hydrochloride, $( \pm)$-Metoprolol, $( \pm)$-Verapamil, Flecainide acetate, $( \pm)$-Sotalol hydrochloride (all dissolved in distilled water) and Amiodaron hydrochloride (dissolved in DMSO). For the experiments the stock solutions were diluted in IMDM.

\section{BRV-Analysis}

The analysis focused on time domain- and nonlinear-methods. The standard deviation of normal-tonormal intervals (SDNN) was considered to be an expression of overall variability. The standard deviation of successive differences (SDSD), which is equivalent to the square root of the mean squared differences of successive normal-to-normal intervals (RMSSD), was rated as a parameter describing the short-term variability $[7,30]$.

It has been reported that the heart rate can influence the calculation of HRV parameters. In order to remove this influence, it is recommended to divide the HRV indices by the corresponding mean normal-tonormal interval [31]. For this reason, the coefficient of variation (CV, SDNN divided by the mean normal-tonormal interval) was included into the analysis of this study.

In order to visualize the BRV, scatterplots (Poincaré-Plots) were generated. In these plots, each time interval between two successive beats (inter-beat interval $\left(\mathrm{IBI}_{n}\right)$ ) is plotted against the subsequent interval $\left(\mathrm{IBI}_{\mathrm{n}+1}\right)$. Accessorily, we added the standard Poincaré-Plot descriptors SD1 and SD2 to the scatterplots. SD1 represents a measure for short-term variability, whereas SD2 describes long-term variability. The descriptors are highly correlated with the time domain parameters [30, 32, 33].

\section{Data Analysis}

The MEA data were analyzed with a customized macro script for the Spike2-software (Cambridge Electronic Design, Cambridge, UK) calculating all parameters mentioned above. With the purpose of eliminating artifacts and outliers, the following exclusion criteria were applied, leading to the results presented in Fig. 2-5:

a) The recording of a single concentration of a measurement that differed in terms of recording time from the corresponding control measurement was excluded (as reasoned in [7]). b) A complete measurement was excluded in case the values for SDNN and SDSD of the lowest measured concentration were $<60 \%$ of the corresponding control measurement. c) The recording of a single concentration of a measurement was excluded in case the value of one of the observed parameters differed by more than two standard deviations from the mean of all measurements with this particular concentration. d) In the case of three or more concentrations of the same measurement meeting criterion c), the complete measurement was excluded.

Data are presented as mean \pm standard error of the mean (SEM). Statistical analysis was accomplished by the Wilcoxon-Test (every concentration compared to control) using SPSS software (IBM, USA). P-values $\leq$ 0.05 were considered statistically significant.

\section{KARGER}




\section{Cellular Physiology Cell Physiol Biochem 2016;38:646-658 and Biochemistry \begin{tabular}{l|l} 
DOI: 10.1159/000438657 2016 The Author(s). Published by S. Karger AG, Basel \\
Published online: February 08, 2016
\end{tabular}

\section{Results}

The mean beating frequency of all measurements $(n=37)$ was $4.1 \pm 0.2 \mathrm{~Hz}$ (range 1.6$7.0 \mathrm{~Hz}$ ). The mean SDNN was $7.0 \pm 1.0 \mathrm{~ms}$, whereas the mean SDSD was $5.5 \pm 0.6 \mathrm{~ms}$. The mean CV was $0.023 \pm 0.002$.

All antiarrhythmic agents, except Verapamil (see below), were tested in a concentration range from $10^{-10} \mathrm{M}$ to $10^{-4} \mathrm{M}$. Our major findings show that Verapamil, Flecainide, Sotalol as well as Ivabradine affected BRV significantly. The effects were dose-dependent. Considering the Poincaré-Plots generated for measurements without pharmacological intervention, three different shapes of scatterplots occurred most frequently (Fig. 1).

\section{Verapamil}

The recordings of the electrical activity of cardiomyocytes within six EBs were included into the analysis (Fig. 2). Verapamil was applied in a concentration range from $10^{-10} \mathrm{M}$ to $10^{-5} \mathrm{M}$. The contracting areas of all EBs that were exposed to $10^{-5} \mathrm{M}$ Verapamil $(\mathrm{n}=5)$ stopped beating during the recording period; four out of five EBs recovered after the washout. Another EB did not exhibit any observable contraction under the microscope after the recording period with $10^{-6} \mathrm{M}$ Verapamil, but also recovered after washout. All parameters describing the BRV increased while the beating frequency decreased at a concentration of $10^{-6} \mathrm{M}$ and $10^{-5} \mathrm{M}$.

\section{Flecainide}

The class IC antiarrhythmic agent Flecainide was applied to seven EBs (Fig. 3 ). Reduced beating frequency occurred at a concentration of $10^{-5} \mathrm{M}(78.0 \pm 4.7 \%$ of control, $\mathrm{p}<0.05)$ in all EBs $(\mathrm{n}=7)$ and at a concentration of $10^{-4} \mathrm{M}(64.7 \pm 8.0 \%$ of control, $p<0.05$ ) in six out of seven EBs. It has to be noted that five out of seven measurements at a concentration of $10^{-4} \mathrm{M}$ Flecainide had to be stopped before the end of the recording period because of decreasing FP amplitude. When $10^{-4} \mathrm{M}$ Flecainide was applied, three EBs exhibited a burst-like beating pattern followed by partial recovery after washout. At the same concentration, another three EBs displayed a much more stable beating pattern compared to the corresponding control measurements, which is also expressed by much lower values for SDSD. After the washout, the values for SDSD and SDNN of these three EBs were clearly higher than the values of the corresponding control measurements. In contrast to the other antiarrhythmic agents that were tested, we found a significantly decreased short-term variability at a concentration of $10^{-9} \mathrm{M}$ Flecainide (SDSD $79.0 \pm 8.7 \%$ of control, $\mathrm{p}<0.05$ ) (Fig. 3). In addition, SDSD was markedly reduced in three EBs at $10^{-7} \mathrm{M}$ as well as $10^{-6} \mathrm{M}$.

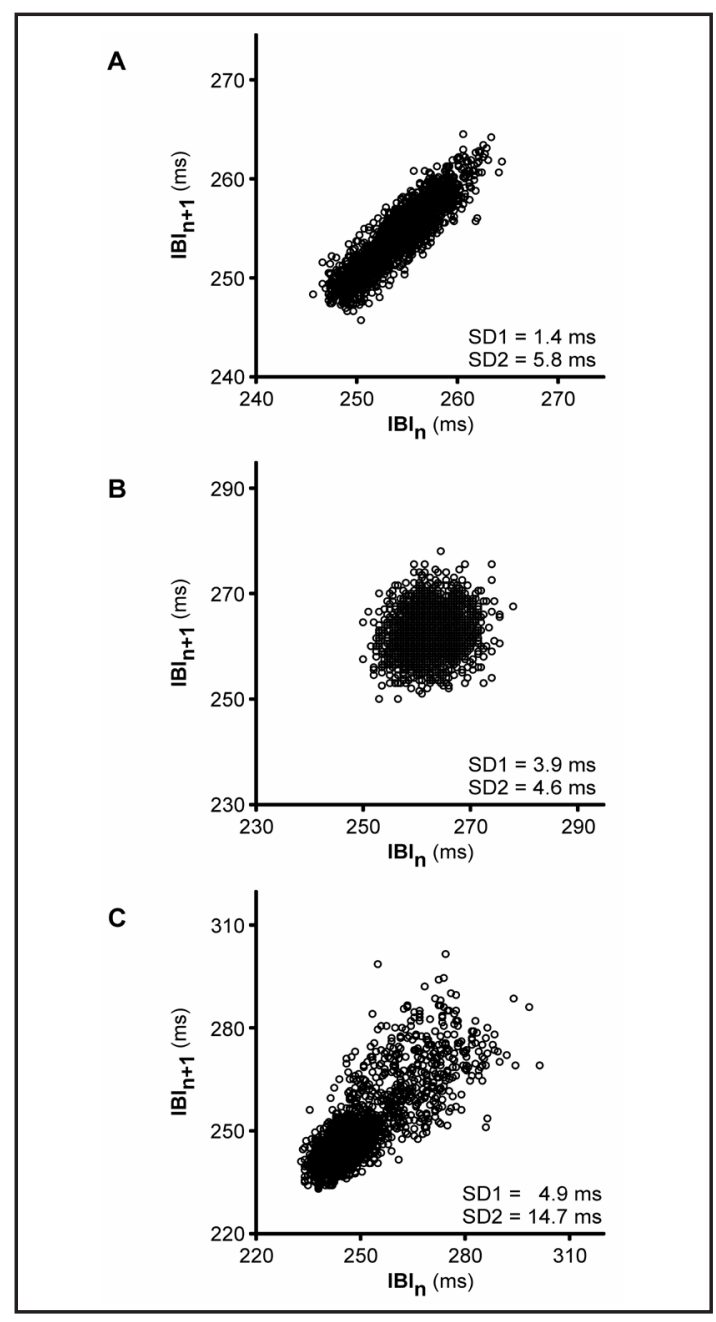

Fig. 1. Poincaré-Plots demonstrating the BRV of mESC-derived cardiomyocytes. (A-C) Typical shapes of Poincaré-Plots generated from measurements without pharmacological intervention (A) Cigarshape (B) Ball-shape (C) Fan-shape. 


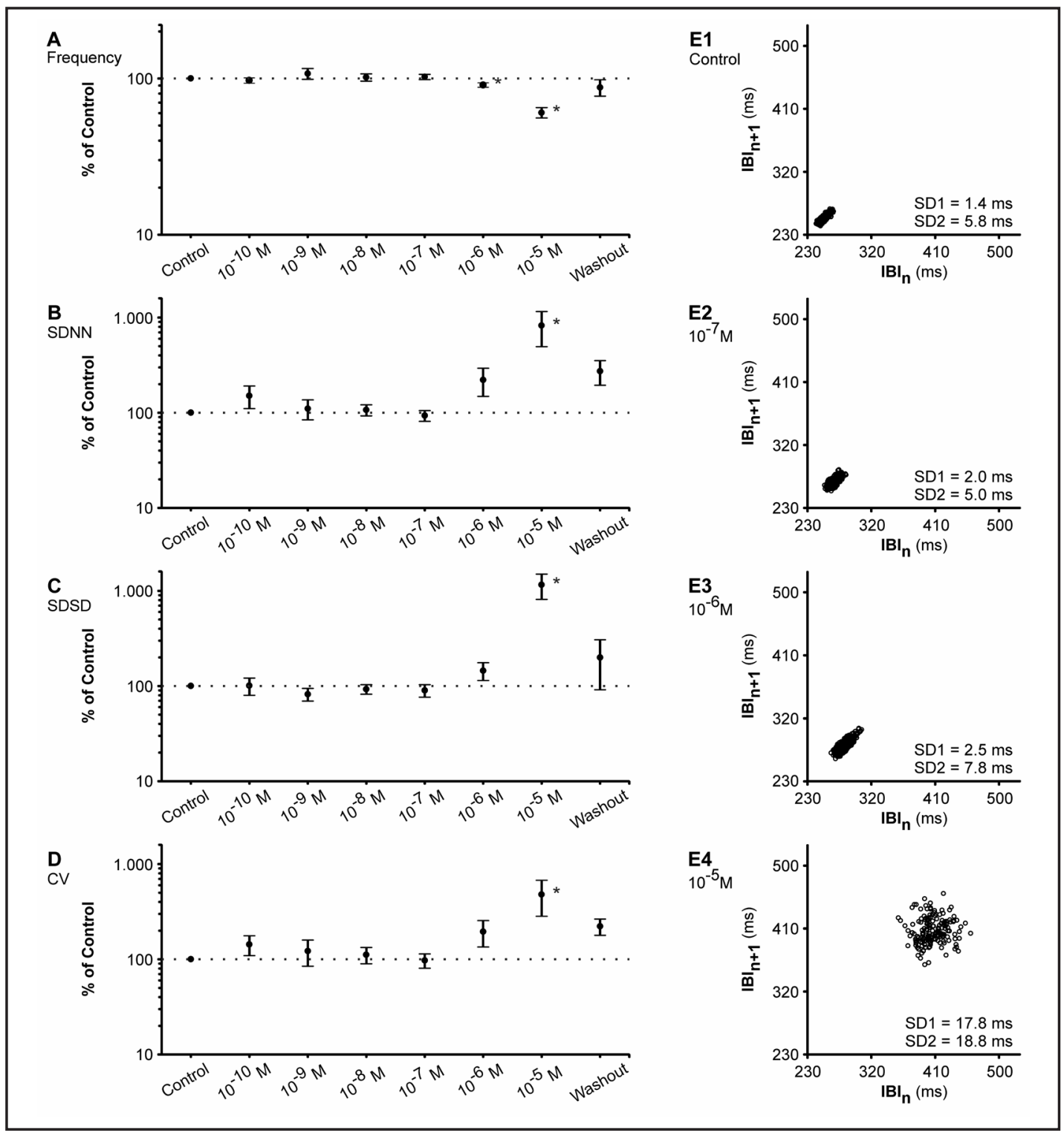

Fig. 2. Effect of Verapamil on BRV $(n=6)$. (A-D) Beating frequency, SDNN, SDSD and CV figured as mean \pm SEM. Verapamil decreased the beating frequency and increased BRV parameters dose-dependently. All EBs that were exposed to $10^{-5} \mathrm{M}$ stopped beating before the end of the recording period $(\mathrm{n}=5)$. E1-4: PoincaréPlots of a representative measurement. E4: The enlargement of the cloud shaped by the IBIs points towards increased variability, whereas the shift of the cloud to the upper right corner of the plot indicates decreased beating frequency. Measurements performed on six EBs $(n=6)$ were included into the analysis. The EBs were derived from three different differentiation cultures. Each EB was measured once. $*$ p $<0.05$.

\section{Sotalol}

Sotalol was tested in seven EBs (Fig. 4). All contracting clusters responded to $10^{-4} \mathrm{M}$ Sotalol with reduced beating frequency $(84.1 \pm 2.8 \%$ of control, $\mathrm{p}<0.05)$ as well as increased over-all variability (SDNN $138.6 \pm 10.3 \%$ of control, $\mathrm{p}<0.05$ ). The CV and the SDSD were not significantly affected by any of the tested concentrations.

\section{Ivabradine}

Six EBs were included into the analysis of the effect of Ivabradine (Fig. 5). Concentrations from $10^{-6} \mathrm{M}$ to $10^{-4} \mathrm{M}$ led to a dose dependent decrease of the beating rate in all contracting 


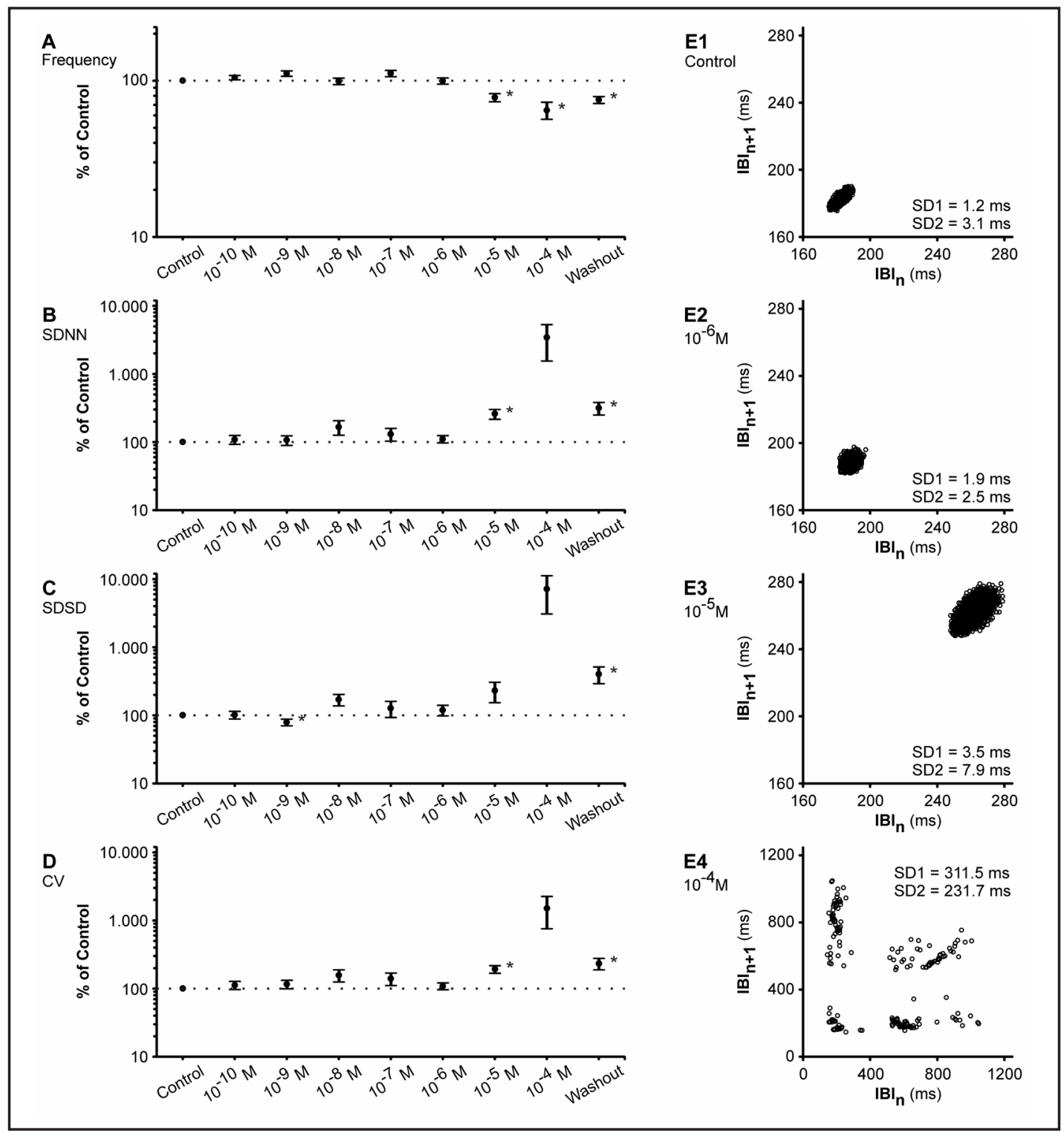

Fig. 3. Effect of Flecainide on BRV ( $n=7$ ). (A-D) Beating frequency, SDNN, SDSD and CV figured as mean \pm SEM. Flecainide induced a decrease in beating frequency and an increase in BRV parameters in a dosedepending manner. The results presented for $10^{-4} \mathrm{M}$ include measurements that had to be terminated before the end of the recording period. E1-4: Poincaré-Plots of a representative measurement. The cloud formed by the IBIs becomes larger and drifts towards the upper right corner of the plot. This indicates lower beating frequency and higher variability. Note the different scaling of the plot in E4 due to the burst-like beating pattern. Measurements performed on seven EBs $(n=7)$ were included into the analysis. The EBs were derived from four different differentiation cultures. Each EB was measured once. ${ }^{*} \mathrm{p}<0.05$.

clusters. The BRV was augmented at a concentration of $10^{-7} \mathrm{M}$ (SDNN $207.6 \pm 66.1 \%$ of control). This effect (increased variability) was even greater at concentrations of $10^{-6} \mathrm{M}$ and $10^{-5} \mathrm{M} .10^{-4} \mathrm{M}$ Ivabradin was applied to four EBs. The recording of the electrical activity of two EBs had to be terminated early. The SDNN as well as the SDSD were reduced in three out of these four EBs in comparison to the preceding concentration. In total, the variability at $10^{-4} \mathrm{M}$ was still higher than in control measurements (Fig. 5). 


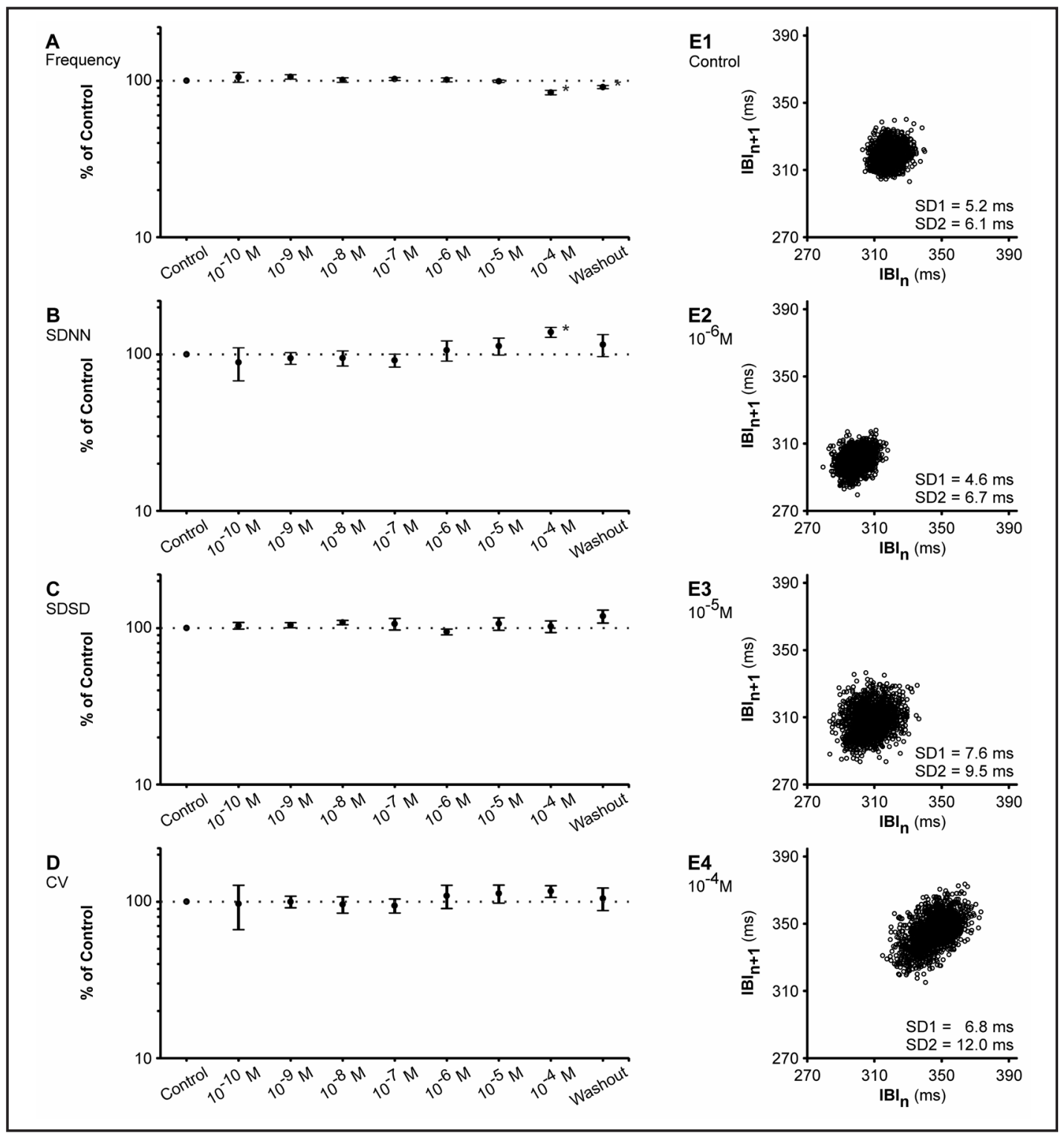

Fig. 4. Effect of Sotalol on BRV ( $n=7$ ). (A-D) Beating frequency, SDNN, SDSD and CV figured as mean \pm SEM. $10^{-4}$ M Sotalol decreased the beating frequency and increased SDNN. E1-4: Poincaré-Plots of a representative measurement. The low impact of Sotalol on BRV and beating frequency can be identified by the relatively unchanged size and position of the clouds shaped by the IBIs in the scatterplots. Measurements performed on seven EBs $(n=7)$ were included into the analysis. The EBs were derived from three different differentiation cultures. Each EB was measured once. * $\mathrm{p}<0.05$.

\section{Metoprolol}

Apart from lowering the beating rate at a concentration of $10^{-4} \mathrm{M}(74.8 \pm 4.7 \%$ of control, $\mathrm{p}<0.05, \mathrm{n}=5$ ), the $\beta$-blocker Metoprolol did not evoke any significant effects on the BRV (data not shown). Nevertheless, there was a trend towards augmented BRV at higher concentrations of Metoprolol.

\section{Amiodarone}

None of the observed parameters describing the BRV was significantly affected by the application of Amiodarone in a concentration range from $10^{-10} \mathrm{M}$ to $10^{-4} \mathrm{M}(\mathrm{n}=5$, data not shown). 


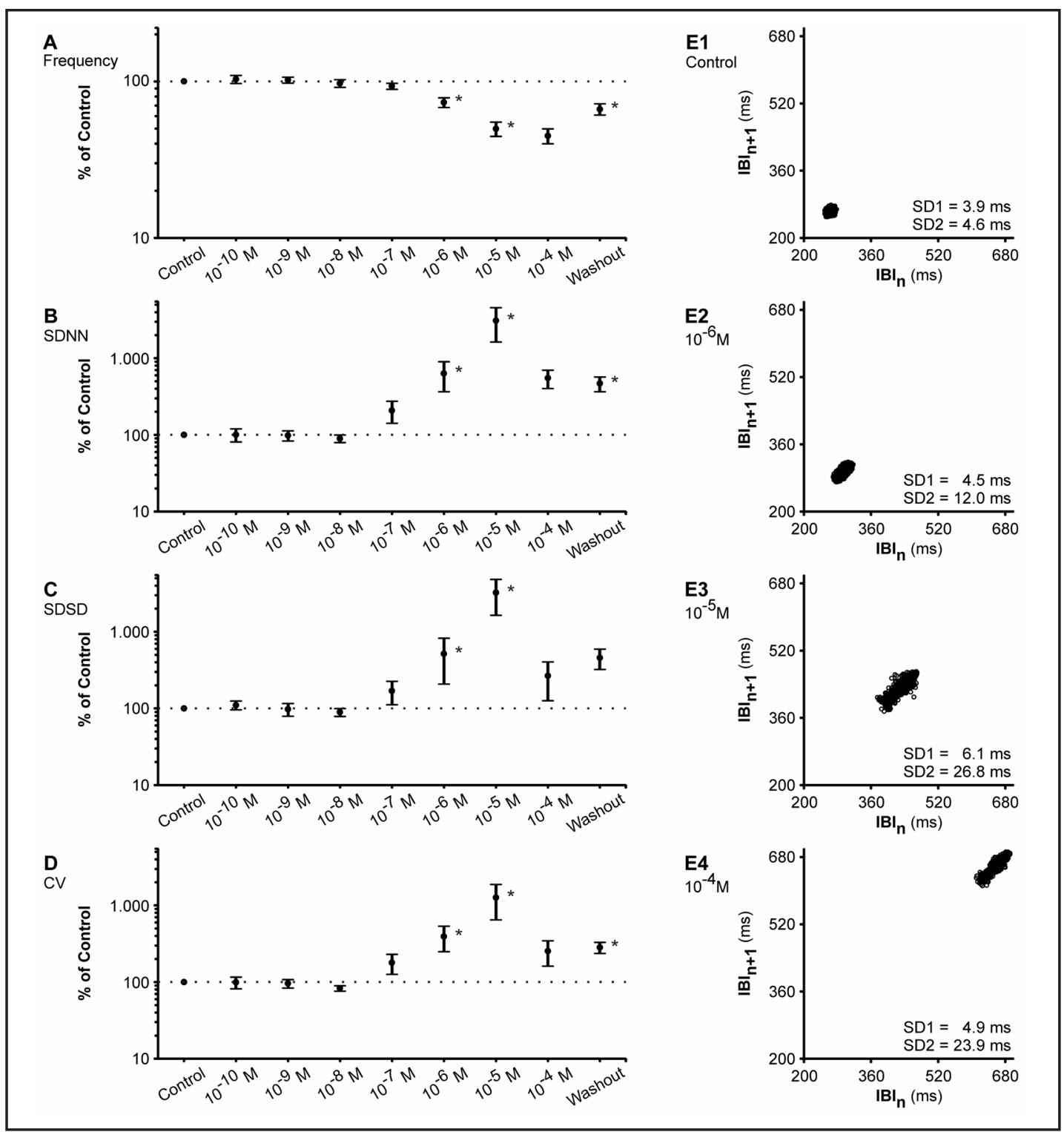

Fig. 5. Effect of Ivabradine on BRV $(n=6)$. (A-D) Beating frequency, SDNN, SDSD and CV figured as mean \pm SEM. Ivabradine decreased the beating frequency and increased BRV parameters dose-dependently. E1-4: Poincaré-Plots of a representative measurement. It is clearly visible that the size of the cloud in E4 is smaller and located further to the top right of the scatterplot compared to E3. This demonstrates visually the lower beating frequency and variability at $10^{-4} \mathrm{M}$ in comparison to $10^{-5} \mathrm{M}$. Measurements performed on six EBs $(n=6)$ were included into the analysis. The EBs were derived from three different differentiation cultures. Each EB was measured once. ${ }^{*} \mathrm{p}<0.05$.

\section{Discussion}

In the present study, we provide evidence for the existence of BRV in cardiomyocytes derived from mESCs and demonstrate that in accordance with clinical observations Verapamil, Flecainide, Sotalol as well as Ivabradine affected BRV significantly. Neither Amiodarone, nor Metoprolol altered BRV parameters significantly.

The MEA technique enabled us to perform stable recordings of the electrical activity of ESC-derived cardiomyocytes. The high sampling rate recordings and subsequently the 


\section{Cellular Physiology Cell Physiol Biochem 2016;38:646-658 \begin{tabular}{l|l|l|l} 
DOI: 10.1159/000438657 & (c) 2016 The Author(s). Published by S. Karger AG, Basel \\
www.karger.com/cpb
\end{tabular} \\ Niehoff et al.: Effect of Antiarrhythmic Drugs on Beat Rate Variability}

offline analysis of the MEA data allowed the investigation of complex physiological processes such as BRV.

Since the HRV has been subject to numerous in vivo studies, different methods for the analysis of the variability have been established in various clinical settings. In terms of Poincaré-Plot analysis, it has been shown that the shape of the scatterplot can be categorized and depends on the medical condition of the patient [33]. The shapes of the scatterplots that occurred most frequently in our in vitro model (Fig. 1), are very similar to shapes that have been described previously in clinical studies [33-35]. Taking into account that the PoincaréPlot analysis can reveal detailed information about HRV and the strong resemblance between in vitro and in vivo scatterplots, this method might be suitable for detecting similarities between the BRV of mESCs-derived cardiomyocytes and the HRV of humans.

Many clinical trials have studied the effects of various antiarrhythmic agents on HRV in patients after acute cardiac events or chronic heart diseases as well as in normal subjects (Table 1).

Increased HRV as a result of treatment with Verapamil has been reported for patients after acute myocardial infarction [14, 21]. The importance of intracellular calcium pathways for the generation of BRV has recently been investigated in human ESC derived cardiomyocytes [6]. Consequently, the augmented BRV as a response to Verapamil in our in vitro model is consistent with clinical observations as well as in vitro studies in other species.

Flecainide has been reported to reduce time domain parameters of HRV in patients suffering from different heart diseases [17-19]. In addition to these findings in adults, case reports have been published which describe reduced fetal HRV after prenatal treatment with Flecainide [36, 37]. In line with these clinical observations, we found a reduction of shortterm variability at comparable concentrations of Flecainide. Nevertheless, as described in the results, not all EBs follow identical trends at these low concentrations. At higher concentrations $\left(\geq 10^{-5} \mathrm{M}\right)$ Flecainide increased BRV markedly. Especially the irreversible effects induced by $10^{-4} \mathrm{M}$ lead to the assumption that Flecainide has reached toxic concentrations. The diverse reactions of the EBs may be explained by pharmacodynamic variability as it has been reported previously or by structural differences within the cell clusters [38].

A clinical trial in patients with ventricular arrhythmias has shown that Sotalol augments time domain specific values of HRV [16]. On one side, we were able to reproduce the increase of variability in terms of SDNN. On the other side, the SDSD remained unchanged and was not affected significantly by any concentration. An explanatory approach might be the pharmacological properties of the drug. Sotalol exhibits characteristics of $\beta$-blockers and class III antiarrhythmic agents [39]. As we show in this study, $\beta$-receptor blockage does not have a striking impact on BRV. So, it is possible to assume that the potassium current, which is also affected by Sotalol, does not contribute much to the generation of BRV.

Clinical studies have documented that the administration of Ivabradine to patients suffering from nonischemic heart failure enhances time domain measures of HRV [15]. At the same time, an in vitro study, using human ESC-derived cardiomyocytes, concluded that $10^{-6} \mathrm{M}$ Ivabradine does not affect BRV [6]. In contrast to the experimental study, we detected a significant increase of all time domain measures when $10^{-6} \mathrm{M}$ Ivabradine was applied. Therefore, the results obtained from our in vitro model, basing on murine ESCderived cardiomyocytes, are in accordance with clinical observations. The effect evoked by the application of $10^{-4} \mathrm{M}$ Ivabradine may be explained by its pharmacological properties. At therapeutic concentrations, Ivabradine binds specifically to the funny-channel, whereas it has been reported to operate less specific, at higher concentrations [40].

The previously published data concerning the effect of $\beta$-blockers on HRV parameters are inconsistent. Depending on the patient population, $\beta$-blockers either increase or do not affect the various time domain parameters. In our in vitro model, Metoprolol did not induce any significant changes in terms of BRV, but still generated a trend towards increased variability. Thus, our results are in accordance with clinical observations. In addition, it is interesting that a high concentration of Metoprolol $\left(10^{-4} \mathrm{M}\right)$ had to be applied in order to lower the beating frequency significantly. However, this particular finding is supported by 


\section{Cellular Physiology Cell Physiol Biochem 2016;38:646-658

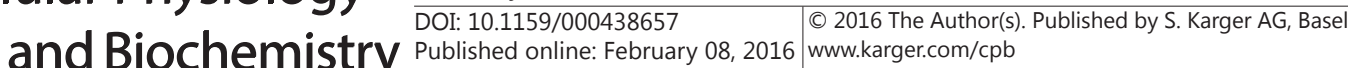

other experimental studies. It has been reported previously, that the application of $\beta$-blockers does not lower the beating frequency of cardiomyocytes derived from induced pluripotent stem cell (iPSC) and human ESCs [41, 42].

The clinical evaluation of the influence of Amiodarone on time domain indexes of HRV in patients with congestive heart failure showed that neither RMSSD, nor SDNN were significantly affected [22]. Another study, focusing on patients with ventricular arrhythmia, was also unable to detect an effect of Amiodarone on HRV [19]. Our experiments cover the therapeutic concentration range of Amiodarone. In accordance with the clinical findings, Amiodarone did not alter BRV parameters significantly in our setting.

\section{Conclusion}

In summary, we demonstrated that mESC-derived cardiomyocytes exhibit BRV, similar to the HRV in humans. In line with clinical observations, antiarrhythmic agents are capable of influencing BRV parameters significantly. Thus, our study emphasizes the potential of this murine ESC-based in vitro model to contribute to the exploration of basic electrophysiological properties as well as to serve as a preclinical screening tool to detect drug effects and side effects.

\section{Limitations}

It should be noted that HRV parameters in clinical settings are usually obtained from long-term recordings and under different circumstances. In addition, species-dependent differences between murine und human ESCs derived cardiomyocytes have been reported previously and should therefore be considered whenever clinical research and in vitro experiments are compared $[2,43]$.

\section{Acknowledgements}

We thank M. Lehmann, E. Nembo, C. Schaefer and J. Semmler for their assistance in this project. We also thank S. Rohani for her support in cell culture work as well as H. Metzner and his staff for technical support.

\section{Disclosure Statement}

The authors declare that they have no conflict of interest.

\section{References}

1 Reppel M, Igelmund P, Egert U, Juchelka F, Hescheler J, Drobinskaya I: Effect of Cardioactive Drugs on Action Potential Generation and Propagation in Embryonic Stem Cell-Derived Cardiomyocytes. Cell Physiol Biochem 2007;19:213-224.

2 Liang H, Matzkies M, Schunkert H, Tang M, Bonnemeier H, Hescheler J, Reppel M: Human and Murine Embryonic Stem Cell-Derived Cardiomyocytes Serve Together as a Valuable Model for Drug Safety Screening. Cell Physiol Biochem 2010;25:459-466.

3 Braam SR, Tertoolen L, van de Stolpe A, Meyer T, Passier R, Mummery CL: Prediction of Drug-Induced Cardiotoxicity Using Human Embryonic Stem Cell-Derived Cardiomyocytes. Stem Cell Res 2010;4:107-116.

4 Reppel M, Pillekamp F, Brockmeier K, Matzkies M, Bekcioglu A, Lipke T, Nguemo F, Bonnemeier H, Hescheler J: The Electrocardiogram of Human Embryonic Stem Cell-Derived Cardiomyocytes. J Electrocardiol 2005;38:166-170. 


\section{Cellular Physiology Cell Physiol Biochem 2016;38:646-658

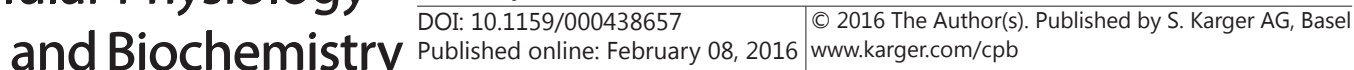 \\ Niehoff et al.: Effect of Antiarrhythmic Drugs on Beat Rate Variability}

5 Mandel Y, Weissman A, Schick R, Barad L, Novak A, Meiry G, Goldberg S, Lorber A, Rosen MR, ItskovitzEldor J, Binah O: Human Embryonic and Induced Pluripotent Stem Cell-Derived Cardiomyocytes Exhibit Beat Rate Variability and Power-Law Behavior. Circulation 2012;125:883-893.

6 Ben-Ari M, Schick R, Barad L, Novak A, Ben-Ari E, Lorber A, Itskovitz-Eldor J, Rosen MR, Weissman A, Binah O: From Beat Rate Variability in Induced Pluripotent Stem Cell-Derived Pacemaker Cells to Heart Rate Variability in Human Subjects. Heart Rhythm 2014;11:1808-1818.

7 Task Force of the European Society of Cardiology and the North American Society of Pacing and Electrophysiology: Heart Rate Variability: Standards of Measurement, Physiological Interpretation and Clinical Use. Circulation 1996;93:1043-1065.

8 Stein PK, Bosner MS, Kleiger RE, Conger BM: Heart Rate Variability: A Measure of Cardiac Autonomic Tone. Am Heart J 1994;127:1376-1381.

9 Kleiger RE, Stein PK, Bigger JT, Jr.: Heart Rate Variability: Measurement and Clinical Utility. Ann Noninvasive Electrocardiol 2005;10:88-101.

10 Pumprla J, Howorka K, Groves D, Chester M, Nolan J: Functional Assessment of Heart Rate Variability: Physiological Basis and Practical Applications. Int J Cardiol 2002;84:1-14.

11 Kleiger RE, Miller JP, Bigger JT, Jr., Moss AJ: Decreased Heart Rate Variability and Its Association with Increased Mortality after Acute Myocardial Infarction. Am J Cardiol 1987;59:256-262.

12 Carpeggiani C, L'Abbate A, Landi P, Michelassi C, Raciti M, Macerata A, Emdin M: Early Assessment of Heart Rate Variability Is Predictive of in-Hospital Death and Major Complications after Acute Myocardial Infarction. Int J Cardiol 2004;96:361-368.

13 Bilchick KC, Fetics B, Djoukeng R, Fisher SG, Fletcher RD, Singh SN, Nevo E, Berger RD: Prognostic Value of Heart Rate Variability in Chronic Congestive Heart Failure (Veterans Affairs' Survival Trial of Antiarrhythmic Therapy in Congestive Heart Failure). Am J Cardiol 2002;90:24-28.

14 Pinar E, Garcia-Alberola A, Llamas C, Vicente T, Lopez-Candel J, Rojo JL, Fernandez R, Valdes M: Effects of Verapamil on Indexes of Heart Rate Variability after Acute Myocardial Infarction. Am J Cardiol 1998;81:1085-1089.

15 Kurtoglu E, Balta S, Karakus Y, Yasar E, Cuglan B, Kaplan O, Gozubuyuk G: Ivabradine Improves Heart Rate Variability in Patients with Nonischemic Dilated Cardiomyopathy. Arq Bras Cardiol 2014;103:308-314.

16 Hohnloser SH, Klingenheben T, Zabel M, Just H: Effect of Sotalol on Heart Rate Variability Assessed by Holter Monitoring in Patients with Ventricular Arrhythmias. Am J Cardiol 1993;72:A67-A71.

17 Bigger JT, Jr., Rolnitzky LM, Steinman RC, Fleiss JL: Predicting Mortality after Myocardial Infarction from the Response of Rr Variability to Antiarrhythmic Drug Therapy. J Am Coll Cardiol 1994;23:733-740.

18 Fauchier L, Babuty D, Autret ML, Poret P, Cosnay P, Fauchier JP: Effect of Flecainide on Heart Rate Variability in Subjects without Coronary Artery Disease or Congestive Heart Failure. Cardiovasc Drugs Ther 1998;12:483-486.

19 Zuanetti G, Latini R, Neilson JM, Schwartz PJ, Ewing DJ: Heart Rate Variability in Patients with Ventricular Arrhythmias: Effect of Antiarrhythmic Drugs. Antiarrhythmic Drug Evaluation Group (Adeg). J Am Coll Cardiol 1991;17:604-612.

20 Lurje L, Wennerblom B, Tygesen H, Karlsson T, Hjalmarson A: Heart Rate Variability after Acute Myocardial Infarction in Patients Treated with Atenolol and Metoprolol. Int J Cardiol 1997;60:157-164.

21 Bonaduce D, Petretta M, Ianniciello A, Apicella C, Cavallaro V, Marciano F: Comparison of Verapamil Versus Felodipine on Heart Rate Variability after Acute Myocardial Infarction. Am J Cardiol 1997;79:564-569.

22 Rohde LE, Polanczyk CA, Moraes RS, Ferlin E, Ribeiro JP: Effect of Partial Arrhythmia Suppression with Amiodarone on Heart Rate Variability of Patients with Congestive Heart Failure. Am Heart J 1998;136:3136.

23 Aronson D, Burger AJ: Effect of Beta-Blockade on Heart Rate Variability in Decompensated Heart Failure. Int J Cardiol 2001;79:31-39.

24 Keeley EC, Page RL, Lange RA, Willard JE, Landau C, Hillis LD: Influence of Metoprolol on Heart Rate Variability in Survivors of Remote Myocardial Infarction. Am J Cardiol 1996;77:557-560.

25 Cook JR, Bigger JT, Jr., Kleiger RE, Fleiss JL, Steinman RC, Rolnitzky LM: Effect of Atenolol and Diltiazem on Heart Period Variability in Normal Persons. J Am Coll Cardiol 1991;17:480-484. 


\section{Cellular Physiology Cell Physiol Biochem 2016;38:646-658

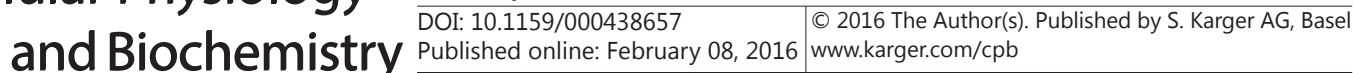 \\ Niehoff et al.: Effect of Antiarrhythmic Drugs on Beat Rate Variability}

26 Kolossov E, Bostani T, Roell W, Breitbach M, Pillekamp F, Nygren JM, Sasse P, Rubenchik O, Fries JW, Wenzel D, Geisen C, Xia Y, Lu Z, Duan Y, Kettenhofen R, Jovinge S, Bloch W, Bohlen H, Welz A, Hescheler J, Jacobsen SE, Fleischmann BK: Engraftment of Engineered Es Cell-Derived Cardiomyocytes but Not Bm Cells Restores Contractile Function to the Infarcted Myocardium. J Exp Med 2006;203:2315-2327.

27 Kolossov E, Lu Z, Drobinskaya I, Gassanov N, Duan Y, Sauer H, Manzke O, Bloch W, Bohlen H, Hescheler J, Fleischmann BK: Identification and Characterization of Embryonic Stem Cell-Derived Pacemaker and Atrial Cardiomyocytes. FASEB J 2005;19:577-579.

28 Reppel M, Pillekamp F, Lu ZJ, Halbach M, Brockmeier K, Fleischmann BK, Hescheler J: Microelectrode Arrays: A New Tool to Measure Embryonic Heart Activity. J Electrocardiol 2004;37:104-109.

29 Hescheler J, Fleischmann BK, Wartenberg M, Bloch W, Kolossov E, Ji G, Addicks K, Sauer H: Establishment of Ionic Channels and Signalling Cascades in the Embryonic Stem Cell-Derived Primitive Endoderm and Cardiovascular System. Cells Tissues Organs 1999;165:153-164.

30 Brennan M, Palaniswami M, Kamen P: Do Existing Measures of Poincare Plot Geometry Reflect Nonlinear Features of Heart Rate Variability? Biomedical Engineering, IEEE Transactions on 2001;48:1342-1347.

31 Sacha J: Interaction between Heart Rate and Heart Rate Variability. Ann Noninvasive Electrocardiol 2014;19:207-2019.

32 Piskorski J, Guzik P: Filtering Poincaré Plots. CMST 2005;11:39-48.

33 Voss A, Schulz S, Schroeder R, Baumert M, Caminal P: Methods Derived from Nonlinear Dynamics for Analysing Heart Rate Variability. Philos Trans A Math Phys Eng Sci 2009;367:277-296.

34 Woo MA, Stevenson WG, Moser DK, Trelease RB, Harper RM: Patterns of Beat-to-Beat Heart Rate Variability in Advanced Heart Failure. Am Heart J 1992;123:704-710.

35 Huikuri HV, Seppanen T, Koistinen MJ, Airaksinen J, Ikaheimo MJ, Castellanos A, Myerburg RJ: Abnormalities in Beat-to-Beat Dynamics of Heart Rate before the Spontaneous Onset of Life-Threatening Ventricular Tachyarrhythmias in Patients with Prior Myocardial Infarction. Circulation 1996;93:18361844.

36 Van Leeuwen P, Schiermeier S, Hailer B, Lange S, Geue D, Hatzmann W, Gronemeyer D: Effect of Prenatal Antiarrhythmic Treatment on Cardiac Function in a Twin Pregnancy. Pacing Clin Electrophysiol 2008;31:1213-1217.

37 van Gelder-Hasker MR, de Jong CL, de Vries JI, van Geijn HP: The Effect of Flecainide Acetate on Fetal Heart Rate Variability: A Case Report. Obstet Gynecol 1995;86:667-669.

38 Padrini R, Piovan D, Busa M, al-Bunni M, Maiolino P, Ferrari M: Pharmacodynamic Variability of Flecainide Assessed by Qrs Changes. Clin Pharmacol Ther 1993;53:59-64.

39 Singh BN, Nademanee K: Sotalol: A Beta Blocker with Unique Antiarrhythmic Properties. Am Heart J 1987;114:121-139.

40 Cargnoni A, Ceconi C, Stavroula G, Ferrari R: Heart Rate Reduction by Pharmacological If Current Inhibition. Adv Cardiol 2006;43:31-44.

41 Yokoo N, Baba S, Kaichi S, Niwa A, Mima T, Doi H, Yamanaka S, Nakahata T, Heike T: The Effects of Cardioactive Drugs on Cardiomyocytes Derived from Human Induced Pluripotent Stem Cells. Biochem Biophys Res Commun 2009;387:482-488.

42 Mehta A, Chung Y, Sequiera GL, Wong P, Liew R, Shim W: Pharmacoelectrophysiology of Viral-Free Induced Pluripotent Stem Cell-Derived Human Cardiomyocytes. Toxicol Sci 2013;131:458-469.

43 Hannes T, Wolff M, Doss MX, Pfannkuche K, Haustein M, Muller-Ehmsen J, Sachinidis A, Hescheler J, Khalil M, Halbach M: Electrophysiological Characteristics of Embryonic Stem Cell-Derived Cardiomyocytes Are Cell Line-Dependent. Cell Physiol Biochem 2015;35:305-314. 